\title{
ON THE STRONG MATRIX SUMMABILITY OF DERIVED FOURIER SERIES
}

\section{K. N. MISHRA and R. S. L. SRIVASTAVA}

Department of Mathematics Indian Institute of Technology Kanpur 208016, India

(Received April 5, 1983, and in revised form August 8, 1983)

ABSTRACT. Strong summability with respect to a triangular matrix has been defined and applied to derived Fourier series yielding a result which extends some known results under a general criterion.

KEY WORDS AND PHRASES. Strong Sumability, Toeplitz matrix, Fourier Series. 1980 MATHEMATICS SUBJECT CLASSIFICATION CODE. $40 F 05$.

1. INTRODUCTION.

The triangular matrix $A=\left[a_{n, k}\right], n, k=0,1, \ldots$ and $a_{n, k}=0$ for $k>n$ is regular if

$$
\begin{aligned}
& \lim _{n \rightarrow \infty} a_{n, k}=0, \\
& \sum_{k=0}^{n}\left|a_{n, k}\right| \leq M, M \text { is independent of } n
\end{aligned}
$$

and

$$
\begin{aligned}
& \qquad \lim _{n \rightarrow \infty} \sum_{k=0}^{n} a_{n, k}=1 \\
& \text { Denoting the sum } \sum_{r=1}^{k} u_{r} \text { by } s_{k} \text {, Fekete [1], defined that the series } \sum u_{r} \text { is }
\end{aligned}
$$
strongly summable to the sum $s$, provided

$$
\sum_{k=1}^{n}\left|s_{k}-s\right|=0(n) \text {. }
$$

This type is now known as strong Cesáro summability of order unity with index 1 or $[C, 1]$ summability.

The series $\Sigma u_{r}$ is said to be strongly summable by Cesáro means, with index $q$, or summable $[C, q]$, or summable $H_{q}$ to the sum $s$ if

$$
\sum_{k=1}^{n}\left|s_{k}-s\right|^{q}=o(n) \text {. }
$$

A special point of interest in the method of summability $\mathrm{H}_{\mathrm{q}}$ lies in the fact that it is given neither by Toeplitz matrix nor by a sequence to function transforma- 
tion. The relationship between summability $H_{q}$ and some regular methods of summation given by $A$ - natrices has been investigated by Kuttner, [2], who proved that if $A$ is any regular Toeplitz method of summability then for any $q(0<q<1)$ there is a series which is not summable $A$ but summable $H_{q}$.

In the present paper we shall define strong summability of series $\Sigma u_{k}$ with the help of a matrix.

DEFINITION. The series $\Sigma u_{k}$ is said to be strongly summable by the regular method $A$ determined by the matrix $\left[a_{n, k}\right]$ with index $q(q>0)$ to the sum $s$ if

$$
\sum_{k=0}^{n} a_{n, k}\left|s_{k}-s\right|^{q}=0(1) \text {, as } n \rightarrow \infty \text {. }
$$

For $a_{n, k}=\frac{1}{n+1}, k \leq n$, we get $(c, 1)$ matrix.

2. MAIN RESULTS.

Let $f(x)$ be a periodic function with period $2 \pi$ and integrable (L) over $(-\pi, \pi)$. Let

$$
f(x) \sim \frac{1}{2} a_{0}+\sum_{1}^{\infty}\left(a_{n} \cos n x+b_{n} \sin n x\right)
$$

be the Fourier series of $f(x)$ and

$$
\sum_{1}^{\infty} n\left(b_{n} \cos n x-a_{n} \sin n x\right)
$$

be the first derived series of (2.1) ottained by term by term differentiation. Write

$$
g(u)=f(x+u)-f(x-u)-2 u f^{\prime}(x),
$$

where $f^{\prime}(x)$ is the derivative of $f(x)$,

$$
G(t)=\int_{0}^{t}|d g(u)| \text {. }
$$

Here we shall take $q=1,2$. Since the case $q=1$ is included in the strong sumability for $q=2$, we omit the same. Precisely we prove the following:

THEOREM. Let $g(u), G(t)$ be defined as in (2.3) and (2.4). If $g(u)$ is a continuous function of bounded variation over $[0, \pi]$ and for some $\beta \geq 1$

$$
G(t)=0\left[t \lambda^{\beta}(t)\right] \text {, as } t \rightarrow 0 \text {, }
$$

where $\lambda^{\beta}(t)$ is a positive function of $t$ such that

$\lambda^{\beta}(t) \rightarrow 0$ as $t \rightarrow 0$,
it is monotonic in $\left(n^{-1}, \delta\right)(\delta$ being small but fixed) and

$$
\int_{n}^{\delta} \frac{\lambda^{2 \beta}(t)}{t} d t=0(1)
$$

then the derived series $(2.2)$ is strongly summable to $f^{\prime}(x)$ by the matrix $(C, 1)$ with index 2 .

Note (2.7) is equivalent to $\frac{\lambda^{2}(t)}{t} \varepsilon L(0, \delta)$. 
In order to prove the theorem we need the following lemma.

LEMMA. If $G(t)=o(t)$ as $t \rightarrow 0$ then for small but fixed $\delta$

$$
\text { (i) } \int_{n}^{\delta} \frac{|d g(t)|}{t} d t \int_{n}^{\delta} \frac{|d g(u)|}{u} d u=o(n)
$$

and

$$
\text { (ii) } \int_{-1}^{\delta} \frac{|d g(t)|}{t^{2}} d t \int_{n}^{t} \frac{|d g(u)|}{u} d u=o(n) \text {. }
$$

PROOF. Since

$$
\begin{aligned}
\int_{n}^{\delta} \frac{\mid d g(u)}{u} \mid d u & =\left[\frac{G(u)}{u}\right]_{n}^{-1}+\int_{n}^{\delta} \frac{G(u)}{u^{2}} d u \\
& =0(1)+\int_{-1}^{\delta} 0\left(\frac{1}{u}\right) d u, \text { in view of }(2.4), \\
& =o(\log n),
\end{aligned}
$$

Therefore

$$
\int_{n}^{\delta} \frac{|d g(t)|}{t} d t \quad \int_{n}^{\delta} \frac{|d g(u)|}{u} d u=o(\log n)^{2}=o(n)
$$

Aga in

$$
\begin{aligned}
\int_{-1}^{\delta} \frac{\mid d g(t)}{t^{2}} d t \int_{n}^{\delta} \frac{|d g(u)|}{u} d u \\
\quad=\int_{n^{-1}}^{\delta} \frac{|d g(t)|}{t^{2}}\left\{\left[\frac{G(u)}{u}\right]_{-1}^{t}+\int_{-1}^{t} \frac{G(u)}{u^{2}} d u\right\} d t \\
=\int_{n}^{\delta} \frac{|d g(t)|}{t^{2}}\left\{\frac{G(t)}{t}+o(1)+o(\log n t)\right\} d t \\
=0(1)\left\{\int_{n}^{\delta} \frac{d g(t)}{t^{2}} \log n t\right\} \\
=0\left\{\left[\frac{G(t)}{t^{2}} \log n t\right]_{n}^{\delta}-\int_{n}^{-1} \frac{G(t)}{t^{3}} d t+2 \int_{n^{-1}}^{\delta} \frac{G(t)}{t^{3}} \log n t d t\right\} \\
=0(n)+0\left(\int_{1}^{n \delta}\left(1 / u^{2}\right) d u\right)+0\left[\int_{1}^{n \delta}\left(\log u / u^{2}\right) d u\right] \\
=0(n) .
\end{aligned}
$$

3. PROOF OF THE THEOREM.

The kth partial sum $\sigma_{k}(x)$ of the series (2.2) is given by [3], 


$$
\sigma_{k}(x)-f^{\prime}(x)=\frac{1}{2 \pi} \int_{0}^{\pi} \frac{\sin (k+1 / 2) t}{\sin \frac{1}{2} t} d g(t) \text {. }
$$

Further, simplifying certain steps as given by [3] and [4] we have

$$
\begin{aligned}
\sigma_{k}(x)-f^{\prime}(x) & =\frac{1}{\pi} \int_{n}^{\pi} \frac{\sin k t}{t} d g(t)+o(1) \\
& =\frac{1}{\pi}\left\{\int_{n}^{\delta}+\int_{\delta}^{\pi}\right\} \frac{\sin k t}{t} d g(t)+o(1) .
\end{aligned}
$$

Therefore

$$
\begin{aligned}
\sum_{k=1}^{n}\left\{\sigma_{k}(x)-f^{\prime}(x)\right\}^{2} & =\frac{1}{\pi^{2}} \int_{-1}^{\delta} \frac{d g(t)}{t} \int_{-1}^{\delta}\left\{\sum_{1}^{n} \sin k t \sin k u\right\} \frac{c g(u)}{u}+o(n) \\
& =\frac{1}{2 \pi^{2}} \int_{-1}^{\delta} \frac{d g(t)}{t} \int_{-1}^{\delta} \sum_{1}^{1}\{\cos k(u-t)-\cos k(u+t)\} \frac{d g(u)}{u}+o(n) \\
& =\frac{1}{2 \pi^{2}} \int_{-1}^{\delta} \frac{d g(t)}{t} \int_{-1}^{\delta} \frac{\sin (n+1 / 2)(u-t)}{2 \sin \frac{1}{2}(u-t)} \frac{1}{u} d g(u) \\
& -\frac{1}{2 \pi^{2}} \int_{-1}^{\delta} \frac{d g(t)}{t} \int_{-1}^{\delta} \frac{\sin (n+1 / 2)(u-t)}{2 \sin \frac{1}{2}(u+t)} \frac{1}{u} d g(u)+o(n) .
\end{aligned}
$$

On simplifying and using the first part of the lemma we obtain

$$
\begin{aligned}
\sum_{k=1}^{n}\left\{\sigma_{k}(x)-f^{\prime}(x)\right\}^{2} & =\frac{1}{2 \pi^{2}} \int_{n}^{\delta} \frac{d g(t)}{t} \int_{n}^{\delta} \frac{\sin n(u-t)}{u(u-t)} d g(u) \\
& -\frac{1}{2 \pi^{2}} \int_{-1}^{\delta} \frac{d g(t)}{t} \int_{n^{-1}}^{\delta} \frac{\sin n(u+1)}{(u+t)} \frac{d g(u)}{u}+o(n) \\
& =P_{1}+P_{2}+o(n), \text { say. }
\end{aligned}
$$

Now, since

$$
\frac{1}{u(u-t)}=\frac{1}{t}\left\{\frac{1}{u-t}-\frac{1}{u}\right\}
$$

and

$$
\int_{n}^{\delta} \frac{d g(t)}{t} \int_{t}^{\delta} \frac{\sin n(u-t)}{u(u-t)} d g(u)=\int_{n}^{\delta} \frac{d g(u)}{u} \int_{n}^{u} \frac{\sin n(u-t)}{t(u-t)} d g(t) .
$$

Therefore

$$
\begin{aligned}
P_{1} & =\frac{1}{2 \pi^{2}} \int_{n}^{\delta} \frac{d g(t)}{t} \int_{-1}^{t} \frac{\sin n(u-t)}{u(u-t)} d g(u)+\frac{1}{2 \pi^{2}} \int_{n}^{\delta} \frac{d g(t)}{t} \int_{t}^{\delta} \frac{\sin n(u-t)}{u(u-t)} d g(u) \\
& =\frac{1}{\pi^{2}} \int_{n}^{\delta} \frac{d g(t)}{t} \int_{-1}^{t} \frac{\sin n(u-t)}{u(u-t)} d g(u)
\end{aligned}
$$




$$
\begin{aligned}
& =\frac{1}{\pi^{2}} \int_{n^{-1}}^{\delta} \frac{d g(t)}{t^{2}} \int_{n^{-1}}^{t}\left(\frac{1}{u-t} \frac{1}{u}\right) \sin n(u-1) d g(u) \\
& =\frac{1}{\pi^{2}} \int_{n^{-1}}^{\delta} \frac{d g(t)}{t^{2}} \int_{n^{-1}}^{t} \frac{\sin n(u-t)}{(u-t)} d g(u)+o\left[\int_{n^{-1}}^{\delta} \frac{|d g(t)|}{t} \int_{-1}^{t} \frac{|d g(u)|}{u}\right] \\
& =\frac{1}{\pi^{2}} \int_{n^{-1}}^{\delta} \frac{d g(t)}{t^{2}} \int_{n}^{t} \frac{\sin n(u-t)}{(u-t)} d g(u)+o(n)
\end{aligned}
$$

by virtue of the second part of the lemma.

Similarly it can be proved that $P_{2}=o(n)$. Thus we get

$$
\sum_{k=1}^{n}\left\{\sigma_{k}(x)-f^{\prime}(x)\right\}^{2}=\frac{1}{\pi^{2}} \int_{n}^{\delta} \frac{d g(t)}{t^{2}} \int_{n}^{t} \frac{\sin n(u-t)}{u(u-t)} d g(u)+o(n) \text {. }
$$

Integration by parts gives

$$
\begin{aligned}
\int_{n}^{t} d g(u) \frac{\sin n(u-t)}{(u-t)}= & {\left[\frac{\sin n(u-t)}{(u-t)} \int_{n^{-1}}^{t} d g(u)\right]_{n}^{-1} } \\
& -\int_{n}^{t}\left[\left\{\frac{n \cos n(u-t)}{(u-t)}-\frac{\sin n(u-t)}{(u-t)^{2}}\right\} d g(u)\right] d u .
\end{aligned}
$$

Using (2.5) this is equal to

$$
\begin{aligned}
{\left[\frac{\sin n(u-t)}{(u-t)} \circ\left\{t \lambda^{\beta}(t)\right\}\right]_{n^{-1}}^{t} } & -o\left[\int_{n}^{t}\left\{n t^{\beta} \lambda(t)\right\} \frac{\cos n(u-t)}{(u-t)} d u\right] \\
& +o\left[\int_{n^{-1}}^{t} \frac{\sin n(u-t)}{(u-t)^{2}}\left\{t \lambda^{\beta}(t)\right\} d u\right] \\
= & o\left[n t \lambda^{\beta}(t)\right] .
\end{aligned}
$$

Therefore

$$
\begin{aligned}
\sum_{k=1}^{n}\left\{\sigma_{k}(x)-f^{\prime}(x)\right\}^{2} & =o\left[n \int_{-1}^{\delta} \frac{d g(t)}{t} \lambda^{\beta}(g)\right]+o(n) \\
& =o(n)\left[G(t) \lambda^{\beta}(t)\right]_{n^{-1}}^{\delta}+o(n)\left[\int_{-1}^{\delta} d g(t) \frac{\lambda^{\beta}(t)}{t^{2}} d t\right] \\
+o(n)\left[\int_{-1}^{\delta} \frac{G(t)}{t}\left\{\beta \lambda^{\beta-1}(t) \lambda^{\prime}(t)\right\} d t\right] & \\
= & o(n)+o(n)\left[\int_{n}^{\delta} \frac{\lambda^{2 \beta}(t)}{t} d t\right] \\
& +o(n)\left[\int_{n}^{\delta} \beta \lambda^{\beta}(t) \lambda^{\beta-1}(t) \lambda^{\prime}(t) d t\right]
\end{aligned}
$$




$$
\begin{aligned}
& =o(n)+o(n)\left[\int_{n^{-1}}^{\delta} \frac{1}{2} \frac{d}{d t}\left\{\lambda^{2 \beta}(t)\right\} d t\right] \\
& =o(n) \text { by the hypothesis }(2.7) .
\end{aligned}
$$

Since $\lambda^{\beta}(t)$ is monotonic, hence its differential coefficient is of constant sign. Thus we get

$$
\sum_{k=1}^{n}\left|\sigma_{k}(x)-f^{\prime}(x)\right|^{2}=o(n)
$$

and therefore

$$
\sum_{k=1}^{n} a_{n, k}\left|\sigma_{k}(x)-f^{\prime}(x)\right|^{2}=o(n) .
$$

This completes the proof of the theorem.

4. SPECIAL CASES.

By way of an application of our theoren, we take $\beta=1, \lambda(t)=1 / \log (1 / t)$ and $a_{n, k}=1$ then the following result follows, [4]:

THEOREM (Shama). At a point for which $f^{\prime}(x)$ exists and

$$
G(t)=o\left[t / \log \frac{1}{t}\right] \text { as } t \rightarrow 0 \text {, }
$$

then

$$
\sum_{k=1}^{n}\left|\sigma_{k}(x)-f^{\prime}(x)\right|^{2}=o(n \log \log n) \text {. }
$$

Since the above theorem is an extension of the result from [C, 1] summability to the case of $[C, 2]$ sumability, (Prasad and Singh [3]), our theorem further extends that result under a general type of criterion.

ACKNOWLEDGEMENT. We are thankful to the referee for his valuable suggestions.

\section{REFERENCES}

1. FEKETE, M. Vizsgalatok a Fourier-Sovokral, Mathematikai es Terniezs Ertesitok 34, (1916), 769-786.

2. KUTTNER, B. Note on Strong Summability, J. Lond. Math. Soc. 21, (1946), 118-122.

3. PRASAD, B. N., and SINGH, U. N. On Strong Sumability of Derived Fourier Series and its Conjugate Fourier Series. Math. $\underline{z}$. 56, 3(1952), 280-288.

4. SHARMA, R. M. On $\mathrm{H}_{2}$ Summability of Derived Fourier Series, Bull. Cal. Math. Soc. 61, (1969), 75-81. 


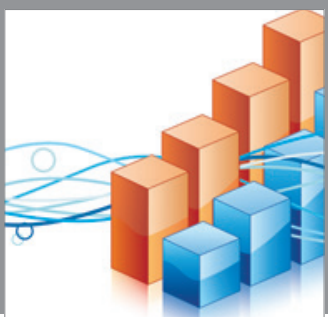

Advances in

Operations Research

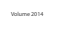

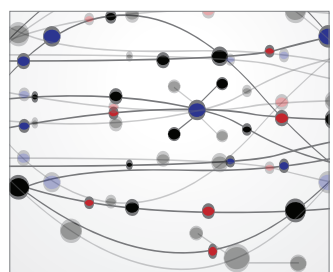

\section{The Scientific} World Journal
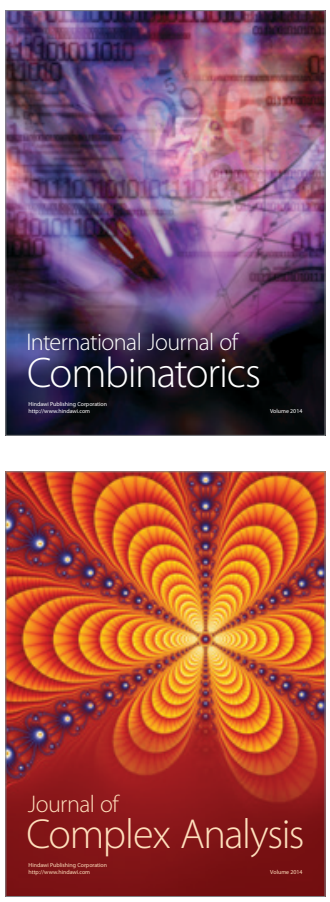

International Journal of

Mathematics and

Mathematical

Sciences
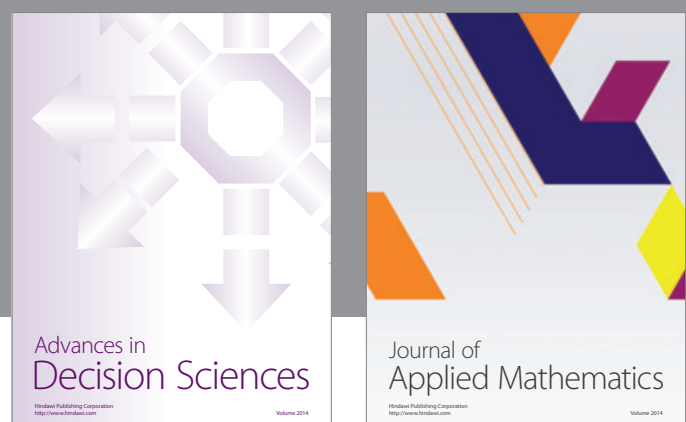

Journal of

Applied Mathematics
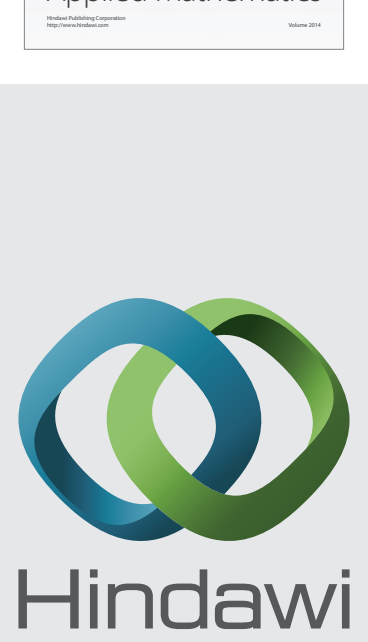

Submit your manuscripts at http://www.hindawi.com
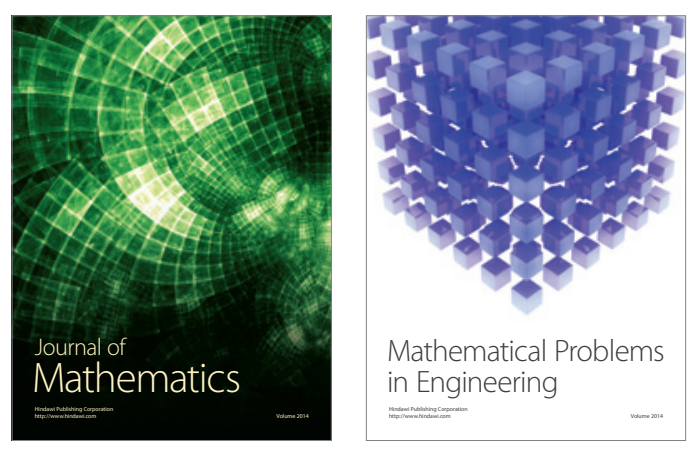

Mathematical Problems in Engineering
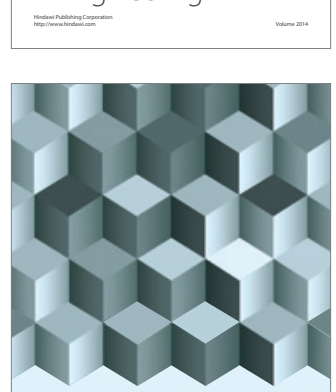

Journal of

Function Spaces
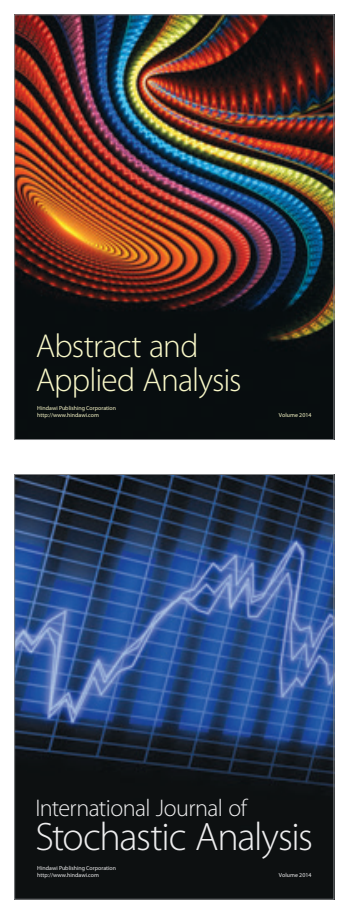

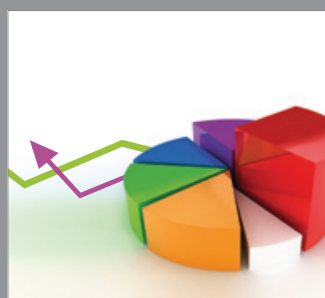

ournal of

Probability and Statistics

Promensencen
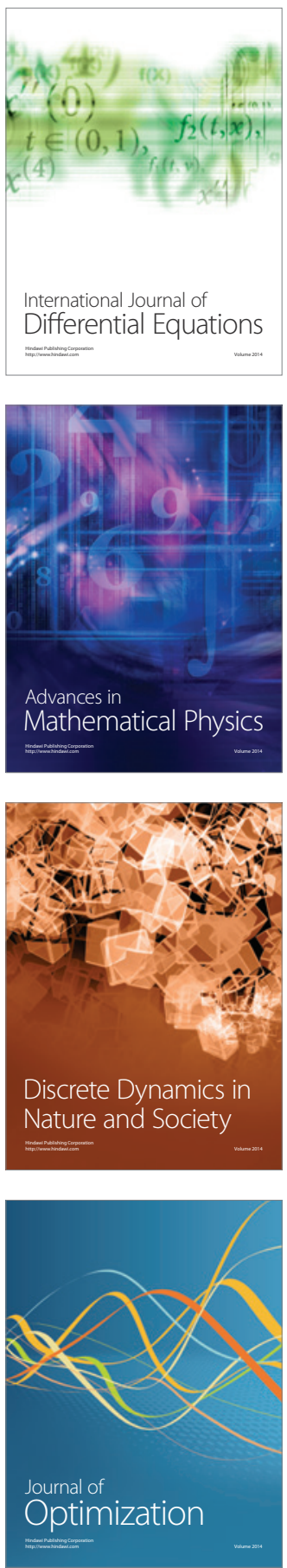